\title{
A case of iliopsoas hematoma as a complication of tetanus in a patient who did not receive anticoagulant therapy
}

\author{
Hiroki Nagasawa ${ }^{*}$ (D), Ken-ichi Muramatsu, Ikuto Takeuchi, Yoshihiro Kushida, Kei Jitsuiki, Jun Shitara, \\ Hiromichi Ohsaka, Kazuhiko Omori, Yasumasa Oode and Youichi Yanagawa
}

\begin{abstract}
Background: The specific clinical feature of tetanus is whole body muscle spasms. These spasms are intensely painful and sometime lead to some injuries. Vertebral fractures have been reported as a common complication of tetanus, however iliopsoas hematoma is a rare complication. We describe a case of iliopsoas hematoma in a tetanus patient who had not been treated with any anticoagulant or antiplatelet agents.

Case presentation: A 72-year-old female patient was transferred to our hospital 7 days after the onset of tetanus. An iliopsoas hematoma was identified in her right iliopsoas muscle on computed tomography. There was no extravasation; thus, the hematoma improved with conservative therapy. There were no episodes that suggested a bleeding tendency, or no factors associated with hemorrhagic conditions.

Conclusion: This is the first report of iliopsoas hematoma as a complication in a tetanus patient who did not received anticoagulation therapy. The possibility of IPH as a complication of tetanus should be considered before and during the administration of anticoagulation therapy.
\end{abstract}

Keywords: Tetanus, lliopsoas hematoma, Complication, Anticoagulation therapy

\section{Background}

Tetanus, a disease that has killed many people since ancient times is caused by tetanospasmin, a toxin produced by Clostridium tetani, which is widely distributed in soil and the human intestines. On the other hand, the neurological prognosis is relatively good. The specific clinical feature of tetanus is whole body muscle spasms (e.g., lock jaw and opisthotonus); these spasms are intensely painful and sometime lead to bone fracture [1].

Common complications of tetanus include hospitalacquired pneumonia (HAP), ventilator-associated pneumonia (VAP), infections (e.g., sepsis), deep vein thrombosis (DVT), pulmonary thromboembolism (PTE) and

\footnotetext{
* Correspondence: hi-nagasawa@juntendo.ac.jp

Department of Acute Critical Care Medicine, Shizuoka Hospital, Juntendo University, 1129 Nagaoka, Izunokuni City, Shizuoka 410-2295, Japan
}

upper gastrointestinal hemorrhage [1, 2]. Vertebral fractures have previously been reported as a complication of opisthotonus [1, 3, 4]. However, iliopsoas hematoma (IPH) is a rare complication of tetanus, with only one report of IPH in a tetanus patient who was treated with anticoagulant therapy to prevent DVT/PTE [5]. We herein describe a case of iliopsoas hematoma in a tetanus patient who had not been treated with any anticoagulant or antiplatelet agents.

\section{Case presentation}

A 72-year-old female patient, who was not prescribed any medications, without any relevant medical history, was transported to our hospital due to suspected tetanus. She had never been vaccinated against tetanus. Fourteen days previously, she had been injured on her

(c) The Author(s). 2020 Open Access This article is licensed under a Creative Commons Attribution 4.0 International License, which permits use, sharing, adaptation, distribution and reproduction in any medium or format, as long as you give appropriate credit to the original author(s) and the source, provide a link to the Creative Commons licence, and indicate if changes were made. The images or other third party material in this article are included in the article's Creative Commons licence, unless indicated otherwise in a credit line to the material. If material is not included in the article's Creative Commons licence and your intended use is not permitted by statutory regulation or exceeds the permitted use, you will need to obtain permission directly from the copyright holder. To view a copy of this licence, visit http://creativecommons.org/licenses/by/4.0/ The Creative Commons Public Domain Dedication waiver (http://creativecommons.org/publicdomain/zero/1.0/) applies to the data made available in this article, unless otherwise stated in a credit line to the data. 
right forearm by a rose thorn while gardening. She did not visit any hospital, and took treated her injury herself. On the 10th day after injury (day 1 ), trismus suddenly appeared. Two days later (day 3) she presented to another hospital with a chief complaint of lock jaw and anorexia, and she was hospitalized for an unknown disease. The muscle tone of her limbs gradually increased after admission, and opisthotonus appeared on days 5-6. Tetanus was suspected, and she was transported to our hospital on day 7 .

On general examination when she arrived at our emergency department, her consciousness was alert. Her vital signs were as follows: blood pressure, 128/88 mmHg; heart rate, 82 beats/minute, respiratory rate, 24 breaths/ minute, and body temperature, $37.9^{\circ} \mathrm{C}$. A physiological examination revealed trismus as small as 2 finger breadths (Fig. 1a), dysphagia, and limb muscle spasm or spinal stiffness that were triggered by stimuli such as light, sounds and a change of body position. The wound

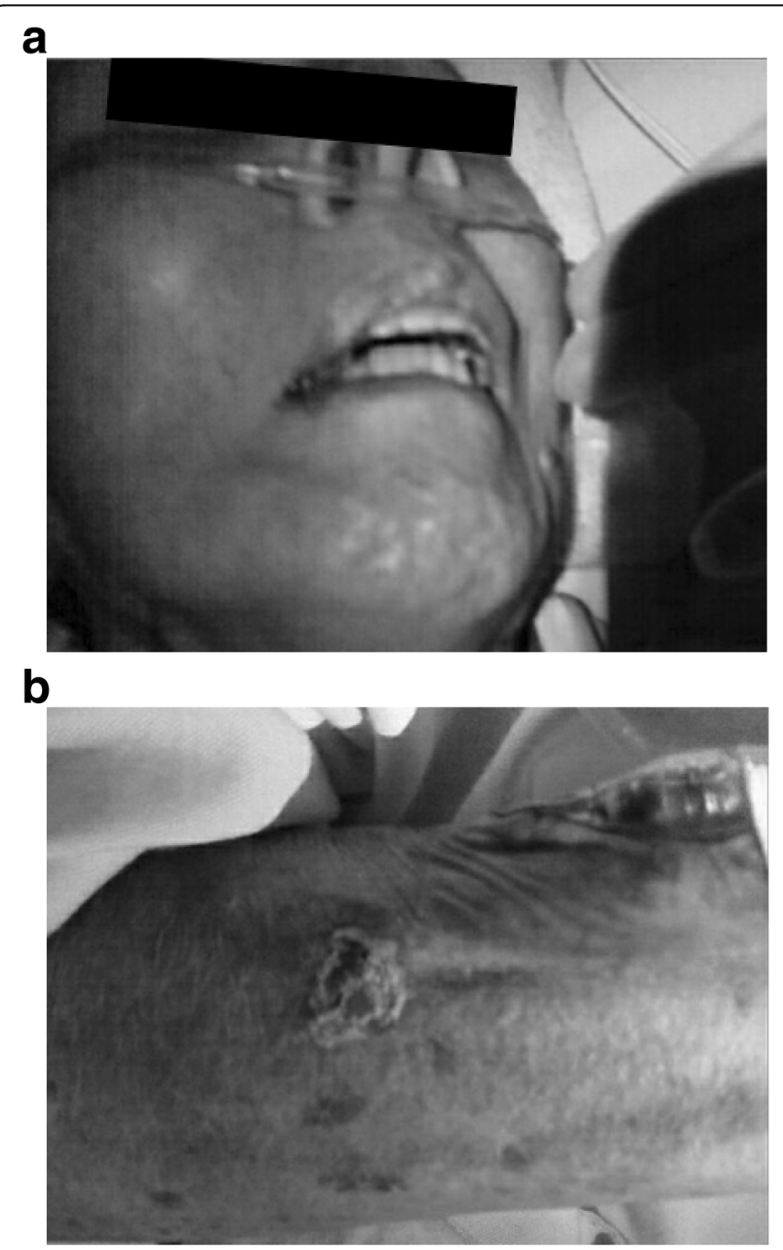

Fig. 1 a A 77-year-old woman with tetanus. Her mouth could not open no more than two finger breadths. b The patient's right forearm was injured by a rose thorn. This wound, which was already covered with scabs, seemed to be the focus of tetanus infection on her right forearm was covered by a scab and no debridement was needed (Fig. 1b).

Whole body computed tomography (CT) was performed on admission and revealed a hematoma of the right iliopsoas muscles without extravasation (Fig. 2b). No hematoma was observed on CT images from a scan performed on day 4 (Fig. 2a). We decided to perform conservative therapy for IPH.

The patient transferred to the intensive care unit (ICU). She was sedated with diazepam and intubated, then mechanical ventilator management was started because she complained of painful spasms. Human tetanus immune globulin (3000 units) was administered intravenously. Penicillin G (8,000,000 units/day) and Ceftriaxone $(2 \mathrm{~g} /$ day $)$ were administered to treat local infection and pneumonia, which was found on CT after her admission. The continuous administration of midazolam and magnesium sulfate hydrate $(50-75 \mathrm{~g} /$ day $)$ was started, and a Kampo medicine, shakuyakukanzoto (7.5 $\mathrm{g} /$ day), was administered by nasogastric tube to relieve the pain caused by her muscle spasms. Muscle relaxants, such as rocuronium and vecuronium, were not required during her hospital stay.

On day 10, we performed tracheostomy in anticipation of the need for long-term mechanical ventilation. On day 17, a CT scan confirmed that the IPH had been absorbed (Fig. 2c), and we started heparin calcium (10, 000 units/day) with subcutaneous injection for the prevention of DVT, where whole-body muscle spasm subsided around day 21, then the mechanical ventilator became dislodged. She was transferred from the ICU to the general medical ward on day 24. During her hospital day, she developed some complications, including HAP/ VAP, catheter-associated infection, methicillin-resistant Staphylococcus aureus pneumonia, cardiopulmonary arrest due to obstruction by sputum; however, all of complications were overcome. The patient underwent rehabilitation in our hospital and was discharged to her home on the day 95.

Prior to the patient's hospitalization, there were no episodes that suggested a bleeding tendency. We performed blood tests to check for any factors associated with hemorrhagic conditions, such as hemophilia; all of these tests were within the normal limits.

\section{Discussion and conclusion}

Tetanospasmin is transferred from the peripheral nerves to the central nervous system, where it inhibits release of glycine and gamma-aminobutyric acid (GABA) and affects the inhibitory motor neurons and autonomic nervous system $[1,2,6]$. These neurons disorders lead to complications such as autonomic dysfunction, arrhythmia, takotsubo cardiomyopathy, cardiac arrest, respiratory failure, ileus, diarrhea and renal failure $[1,2$, 


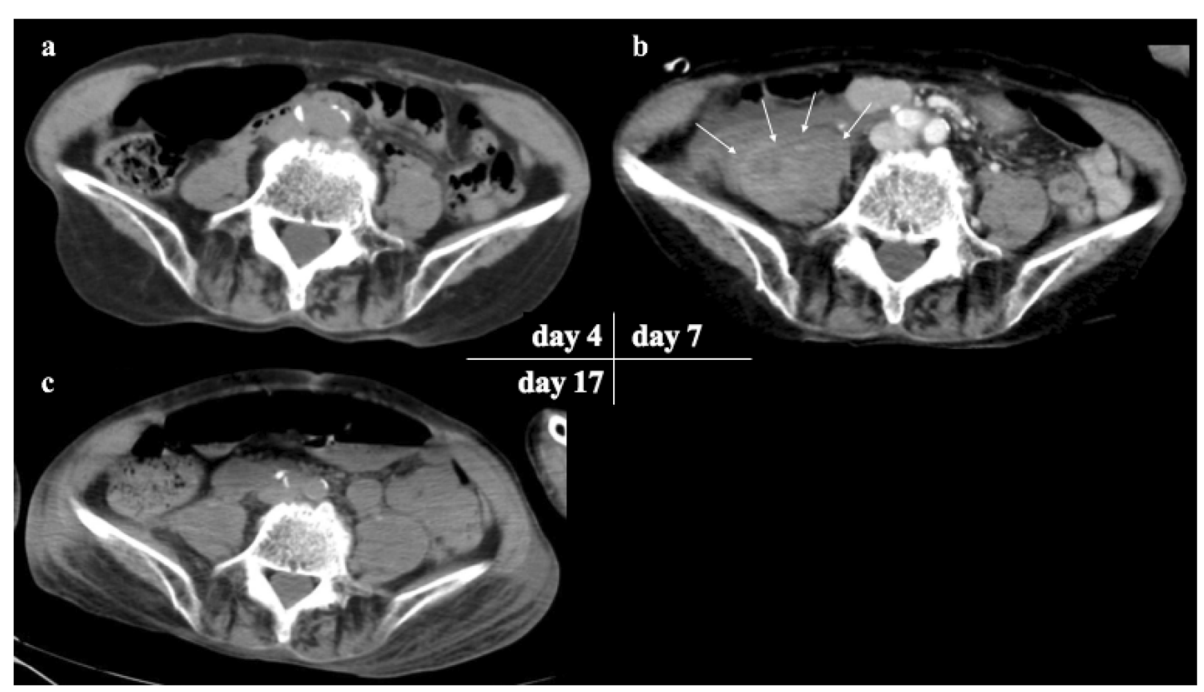

Fig. 2 There was no hematoma on day 4 (a). Right iliopsoas hematoma was unexpectedly found without extravasation on day 7. White arrows $(\downarrow)$ indicate hematoma in the right iliopsoas muscle (b). After conservative treatment, the iliopsoas hematoma become unclear on day 17 (c)

7]. In some previous reports vertebral fractures was reported in patients with severe opithotonus $[1,3,4]$.

Ishii et al. reported that 4 of $21(19 \%)$ patients with tetanus developed iliopsoas hematoma as a complication during their clinical course [5]. Iliopsoas hematoma is reported to occur in $0.3 \%$ of ICU patients [8,9]; thus, the rate of $19 \%$ seems high. No reports have suggested a relationship between tetanus and IPH, and this is the first report to demonstrate that IPH may be a complication of tetanus. In the report by Ishii et al., all 4 cases with IPH received anticoagulation therapy. Thus, it was not possible to determine whether tetanus or anticoagulation therapy was the main cause of IPH [5].

Commonly reported risk factors for IPH include trauma, anticoagulation therapy, and hemodialysis [810]. In our report, patient, CT scans obtained on day 4, when opisthotonus appeared, showed no sign of IPH; however, hemorrhage was observed in the right iliopsoas muscle on day 8 , before the administration of heparin. This patient's body had not been injured and she was not a dialysis patient. Furthermore, we did not detect any underlying disease associated with a bleeding tendency. From these results, we diagnosed the IPH as a complication of tetanus.

The mortality rate of IPH in the ICU has been reported to be $30-50 \%[8,9]$. On the other hand, in all five of the reported cases of IPH with tetanus, including those reported by Ishii et al. [5], the patient survived; thus, IPH with tetanus may not affect the outcomes.

In the management of severe tetanus, sedation and muscle relaxants are routinely required; thus, it may be difficult to notice the patient's complaint. If our patient had shown back pain, we might incorrectly have concluded that it had been caused by opisthotonus. IPH was found by chance in the present case, without any factors suggestive of bleeding (e.g., hypotension or decreased hemoglobin). Thus, physicians should pay attention to the possible occurrence of IPH in tetanus patients before or during the administration of anticoagulation therapy.

In Japan, penicillin is still the first choice for antibiotics to treat tetanus [11], and some reports have described the use of penicillin in the treatment of tetanus $[7,12]$. Metronidazole infusion was introduced in Japan in 2014; however, its use in tetanus treatment is not covered by insurance. One report noted that the doses of muscle relaxants and sedative required by tetanus patients were lower in those who received metronidazole [13]. In the present case, we conventionally selected penicillin, however, the duration of her illness might have been improved if she was treated with metronidazole instead of penicillin.

In Japan, the incidence of tetanus among young people has decreased since the introduction of the diphtheriatetanus-pertussis (DTP) vaccine; however, tetanus morbidity remains a problem among patients $>60$ years of age [14]. It should be kept in mind that "prevention" - in particular booster vaccination in adults -is of the utmost importance in the management of tetanus.

This is the first report to describe IPH as a complication of tetanus in a patient who was not receiving anticoagulation therapy. It is important to prevent thrombosis in tetanus patients; however, the possibility of IPH as a complication of tetanus should be considered before and during the administration of anticoagulation therapy. 


\section{Abbreviations}

CT: Computed tomography; DTP: Diphtheria-tetanus-pertussis; DVT: Deep vein thrombosis; GABA: Glycine and gamma-aminobutyric acid; HAP: Hospital-acquired pneumonia; ICU: Intensive care unit; IPH: Iliopsoas hematoma; PTE: Pulmonary thromboembolism; VAP: Ventilator-associated pneumonia

\section{Acknowledgements}

Not applicable.

\section{Authors' contributions}

$\mathrm{HN}$ : drafting and revision of the manuscript. KM, IT, YK, KJ, JS, HO and YO: discussion and revision of the manuscript. $K O$ and $Y Y$ : final approval of the revised manuscript after correction based on several academic advices for submission. The authors read and approved the final manuscript.

\section{Funding}

The drafting of this manuscript was financially supported by the Ministry of Education, Culture, Sports, Science and Technology (MEXT)-Supported Program for the Strategic Research Foundation at Private Universities, 20152019 concerning [The constitution of total researching system for comprehensive disaster, medical management, corresponding to wide-scale disaster]. The funds are used for payment of English correction fees and posting fees.

\section{Availability of data and materials}

The datasets used during the current study are available from the corresponding author on reasonable request.

\section{Ethics approval and consent to participate}

Not applicable.

\section{Consent for publication}

Written informed consent was obtained from the patient and her family for publication of this case report and photographs.

\section{Competing interests}

The authors declare that they have no competing interests.

Received: 25 April 2020 Accepted: 24 September 2020

Published online: 07 October 2020

\section{References}

1. Cook TM, Protheroe RT, Handel JM. Tetanus a review of the literature. $\mathrm{Br} J$ Anaesth. 2001;87:477-87.

2. Farrar JJ, Yen LM, Cook T, Binh N, Parry J, Parry CM. Tetanus. J Neurol Neurosurg Psychiatry. 2000:69(3):292-301.

3. Davis PR, Rowland HA. Vertebral fractures in west Africans suffering from tetanus: a clinical and osteological study. J Bone Joint Surg Br. 1965;47:61-71.

4. Wilson TJ, Orringer DA, Sullivan SE, Patil PG. An L-2 burst fracture and cauda equina syndrome due to tetanus. J Neurosurg Spine. 2012;16:82-5.

5. Ishii N, Mochizuki H, Shiomi K, Nakazato M. Iliopsoas hematoma as a complication of tetanus. Neurol Clin Neurosci. 2018:1-5. https://doi.org/10. 1111/ncn3.12241.

6. Bleck TP. Pharmacology of tetanus. Clin Neuropharmacol. 1986;9(2):103-20.

7. Akira T, Iwanami N, Yamazaki Y. Severe tetanus complicated by Takotsubo cardiomyopathy. Intern Med. 2019;58:2107-12

8. Artzner T, Clere-Jehl R, Schenck M, Greget M, Merdji H, Marini PD, et al. Spontaneous llio-psoas hematomas complicating intensive care unit hospitalizations. PLoS One. 2019;14(2):e0211680.

9. Llitjos JF, Daviaud F, Grimaldi D, Legriel S, Georges JL, Guerot E, et al. Iliopsoas hematoma in the intensive care unit: a multicentric study. Ann Intensive Care. 2016;6(1):8.

10. Chan YC, Morales JP, Reidy JF, Taylor PR. Management of spontaneous and iatrogenic retroperitoneal haemorrhage: conservative management, ebndovascular intervention or open surgery? Int J Clin Pract. 2008;62(10): 1604-13.

11. Japanese Society of Chemotherapy Committee on guidelines for treatment of anaerobic infections; Japanese Association for Anaerobic Infections
Research. Chapter 2-12-4. Anaerobic infections (individual fields): tetanus. J Infect Chemother. 2011;17 Suppl 1:125-32.

12. Akane $Y$, Tsugawa $T$, Hori $T$, Togashi A, Yoto $Y$, Inazawa $N$, et al. Tetanus in a partially immunized child. J Infect Chemother. 2018;24(12):980-2.

13. Yen LM, Dao LM, Day NPJ. Management of tetanus:a comparison of penicillin and metronidazole. In: Symposium of antimicrobial resistance in southern Viet Nam; 1997.

14. Nakajima M, Aso S, Matsui H, Fushimi K, Yasunaga H. Clinical features and outcomes of tetanus: analysis using a National Inpatient Database in Japan. J Crit Care. 2018;44:388-91.

\section{Publisher's Note}

Springer Nature remains neutral with regard to jurisdictional claims in published maps and institutional affiliations.
Ready to submit your research? Choose BMC and benefit from:

- fast, convenient online submission

- thorough peer review by experienced researchers in your field

- rapid publication on acceptance

- support for research data, including large and complex data types

- gold Open Access which fosters wider collaboration and increased citations

- maximum visibility for your research: over $100 \mathrm{M}$ website views per year

At $\mathrm{BMC}$, research is always in progress.

Learn more biomedcentral.com/submissions 